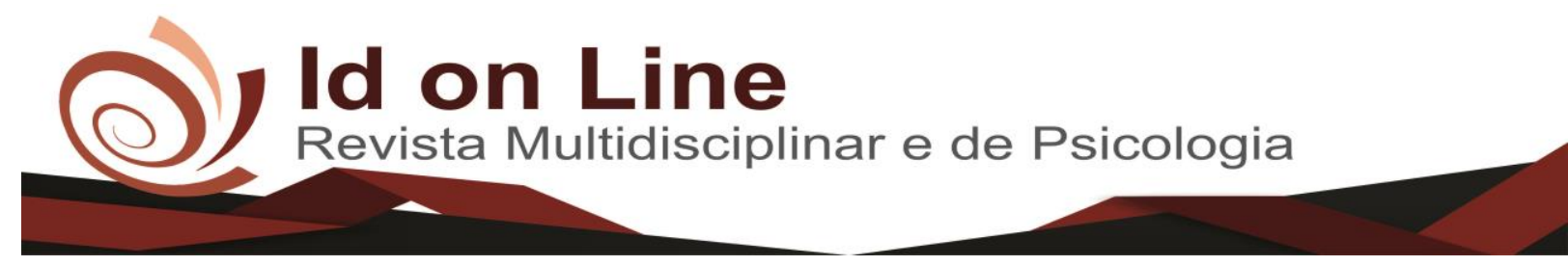

Artigo de Revisão

\title{
Efeito da Drenagem Linfática no Tratamento do Fibro Edema Gelóide em Mulheres
}

\author{
Flávia Ferraz Gusmão $^{1}$; Marcia Meira Guimarães ${ }^{2}$; Natália Cristina Lima Silva ${ }^{3}$; \\ Juliana Barros Ferreira ${ }^{4}$
}

\begin{abstract}
Resumo: O fibro edema gelóide, é classificado como um distúrbio no metabolismo do tecido subcutâneo, que leva a uma alteração no corpo das mulheres e como terapêutica de tratamento, tem-se a drenagem linfática manual. Objetivo: Analisar a eficácia da drenagem linfática manual no tratamento do fibro edema gelóide em mulheres. Metodologia: Foram utilizados artigos científicos em português e inglês, entre os anos de 2010 a 2018. Resultados: Foram encontrados 7 artigos obtidos pela análise de dados, que foram categorizados em um quadro contendo autor/ano, título do estudo, objetivo e resultados. Conclusão: Ficou evidente a eficácia da drenagem linfática manual no tratamento do fibro edema gelóide em mulheres. Porém, observa-se que esta técnica deve ser associada a um conjunto de adaptações de hábitos de vida objetivando um melhor prognóstico da disfunção estética.
\end{abstract}

Palavras Chave: Celulite. Drenagem Linfática Manual. Estética. Imagem Corporal. Saúde.

\section{Effect of the Lymphatic Drainage on Treatment Fibroid Edema in Women}

\begin{abstract}
The fibroid edema geloid is classified as a disorder in the metabolism of subcutaneous tissue, which leads to a change in the body of women and as treatment therapy, we have manual lymphatic drainage. Objective: To analyze the efficacy of manual lymphatic drainage in the treatment of fibrous edema in women. Methodology: Scientific articles were used in Portuguese and English, between the years 2010 to 2018. Results: We found 7 articles obtained by data analysis, which were categorized in a table containing author / year, study title, objective and results. Conclusion: The efficacy of manual lymphatic drainage was evident in the treatment of fibroid edema geloid in women. However, it is observed that this technique must be associated to a set of adaptations of life habits aiming at a better prognosis of aesthetic dysfunction.
\end{abstract}

Keywords: Cellulite. Manual lymphatic drainage. Aesthetics. Body image. Health.

\footnotetext{
${ }^{1}$ Acadêmica do curso Tecnólogo em Estética e Cosmética da Faculdade Independente do Nordeste- FAINOR/Vitória da Conquista, BA. Rua, Luís Eduardo Magalhães, nº1485, Candeias; e-mail: flaviaferraz_14@ hotmail.com

${ }^{2}$ Fisioterapeuta. Mestre em Desenvolvimento Regional e Meio Ambiente/UESC. Docente da Faculdade Independente do Nordeste. FAINOR/Vitória da Conquista, BA, Brasil. Avenida Deputado Luiz Eduardo Magalhães 1305, Bairro Candeias; email:marciameira@fainor.com.br

${ }^{3}$ Acadêmica do curso Tecnólogo em Estética e Cosmética da Faculdade Independente do Nordeste- FAINOR/Vitória da Conquista, BA. Rua, Jovita Lemos, n³00, Candeias; e-mail: naty.cls2@ hotmail.com

${ }^{4}$ Fisioterapeuta. Mestre em Tecnologias em Saúde/EBMSP. Docente da Faculdade Independente do Nordeste FAINOR/Vitória da Conquista, BA, Brasil. Rua SifredoPedral Sampaio, nº414, B. Recreio; e-mail: julianabarros@fainor.com.br
} 


\section{Introdução}

Nos dias atuais existe uma busca contínua pelo corpo perfeito e tão sonhado (FERREIRA, LEMOS, SILVA, 2016). A mídia tecnológica e suas restrições tendem a serem parâmetros que influenciam ambos os sexos (BRITO, SILVA, 2017). No entanto, essas limitações afetam diretamente as mulheres quando tendem a seguir os padrões de beleza impostos pela sociedade (FROIS, MOREIRA, STENGEL, 2011).

E dentre esses indicadores, a maior queixa entre as mulheres está ligado ao fibro edema gelóide (FEG), conhecido popularmente como celulite. Essa disfunção estética atinge em média 85 a 98\% das mulheres de todas as raças (COSTA, 2012). Estudos mostram que entre os sexos há diferença nas características anatômicas e histológicas, das quais existe comprovação que há maior incidência de celulite no sexo feminino (SILVA, et al., 2017).

O FEG é classificado como um distúrbio no metabolismo do tecido subcutâneo caracterizado por uma infiltração edematosa que desencadeia a polimerização da substância fundamental, e após infiltração, resulta uma reação fibrótica consecutiva (CUNHA, CUNHA, MACHADO, 2015). Assim, os mucopolissacarídeos integrados sofrem o processo de geleificação (MENEZES, SILVA e RIBEIRO, 2009). Além disso, a disfunção apresenta alteração visual no relevo cutâneo, que é resultado de modificações estruturais nos adipócitos, e na microcirculação (MATEUS, 2014).

O surgimento do FEG tem se tornado um fator alarmante, visto que ele é uma consequência ocasionada por vários fatores (NOOTHETI, 2006; PETERSON, GOLDMAN, 2011). Entre eles estão: o sedentarismo, estresse, obesidade e estilo de vida (BRANDAO et al, 2010). Por se tratar de uma disfunção multifatorial, para que o tratamento resulte em bons resultados é preciso uma avaliação bem descrita, anamnese e exame físico (NOOTHETI, 2006; PETERSON e GOLDMAN, 2011).

O FEG apresenta três classificações que diferem de acordo as alterações histológicas (VOLPI, 2010). Sendo eles: grau I - para visualização, pressiona o tecido com os dedos o tecido, ou solicita uma contração muscular voluntária; grau II - a alteração tecidual é visível mesmo sem a compressão dos tecidos; grau III - o acometimento tecidual é visualizado com o indivíduo em qualquer posição; e o grau IV - apresenta as mesmas características do grau III, associadas aos nódulos palpáveis, visíveis e dolorosos (PETERSON e GOLDMAN, 2011). 
Pode ser ainda classificado em compacta ou dura, flácida, mista e edematosa quanto a sua forma clínica (GALVÃO, 2005).

A literatura traz diversos recursos estéticos na terapêutica do FEG como: aplicação de dermocosméticos, recursos da eletroterapia e a drenagem linfática manual (DLM) (ALMEIDA et al, 2011). Esta terapêutica tem o objetivo de drenar o excesso de fluído acumulado nos meios intersticiais, e é executada de maneira suave, de forma lenta e no sentido dos vasos linfáticos (RHEUMATOL, 2009; DE MELO CORTEZ, 2013). O tratamento facilita as trocas gasosas e nutricionais no organismo, ajuda a remoção de líquidos ricos em proteínas e toxinas, assim normaliza o potencial hidrogeniônico $(\mathrm{Ph})$ intersticial, favorece a nutrição e a oxigenação tissular (BRANDAO et al, 2010; ABE e FERREIRA, 2014).

Embora estudos não controlados, e sem seguimento de follow up, mostram resultados positivos da DLM para redução do FEG, faz-se necessário estudos com bom delineamento para mostrar a importância deste tratamento (LEDUC, 2007). Diante do exposto, através desta revisão de literatura, objetiva-se analisar a eficácia da drenagem linfática manual no tratamento do fibro edema gelóide em mulheres.

\section{Metodologia}

Trata-se de uma revisão bibliográfica com análise descritiva, onde os resultados foram transcritos de forma dissertativa. Foram utilizados artigos científicos nos idiomas português e inglês. As bases de dados utilizadas foram SCIELO, e o Google Acadêmico, e as seguintes palavras Chave: Celulite. Drenagem Linfática Manual. Estética. Imagem Corporal. Saúde, publicados entre os anos de 2010 a 2018. Foram encontrados 183 artigos, 176 artigos foram descartados, e 7 artigos foram selecionados por se enquadrarem nos critérios de inclusão do estudo. A amostragem da pesquisa seguiu os seguintes critérios de inclusão: artigos sobre os efeitos da drenagem linfática no fibro edema gelóide, disponíveis na íntegra. Critérios de exclusão: artigos que utilizaram tratamentos eletroterápicos, ou dermocosméticos, associados com a drenagem linfática. $\mathrm{O}$ estudo deu-se a partir dos seguintes argumentos: Leitura seletiva (leitura das partes mais importantes de cada artigo) e registro de informações retiradas das fontes. Em seguida foi feito um quadro sobre os artigos relacionados aos efeitos da drenagem linfática no fibro edema gelóide (Quadro 1). 


\section{Resultados}

Foram localizados 183 artigos na base de dados do Scientific Electronic Library Online (SciELO) e Google Acadêmico. Após leitura, apenas 7 artigos foram categorizados no quadro abaixo por estarem de acordo com os critérios de inclusão desse estudo.

Quadro 1: Características dos estudos selecionados sobre Efeitos da drenagem linfática no tratamento do fibro edema gelóide em mulheres.

\begin{tabular}{|c|c|c|c|}
\hline $\begin{array}{c}\text { Autor/ } \\
\text { ano }\end{array}$ & $\begin{array}{c}\text { Título do } \\
\text { estudo }\end{array}$ & Objetivo & Resultados \\
\hline $\begin{array}{c}\text { Brandão et } \\
\text { al., } 2010\end{array}$ & $\begin{array}{l}\text { Avaliação da } \\
\text { técnica de } \\
\text { drenagem linfática } \\
\text { manual no } \\
\text { tratamento do } \\
\text { fibroedema gelóide } \\
\text { em mulheres. }\end{array}$ & $\begin{array}{l}\text { Identificar o efeito da } \\
\text { drenagem linfática } \\
\text { manual (DLM) no } \\
\text { tratamento do fibro } \\
\text { edema gelóide (FEG) em } \\
\text { mulheres. }\end{array}$ & $\begin{array}{l}\text { Após } 10 \text { sessões de DLM, foi } \\
\text { identificado satisfação nas pacientes, } \\
\text { podendo visualizar melhora clínica no } \\
\text { aspecto de pele, tanto através das } \\
\text { fotografias quanto pela inspeção visual. }\end{array}$ \\
\hline $\begin{array}{l}\text { Lima et al., } \\
2010\end{array}$ & $\begin{array}{l}\text { A eficácia da } \\
\text { drenagem linfática } \\
\text { manual para o } \\
\text { tratamento do fibro } \\
\text { edema gelóide. }\end{array}$ & $\begin{array}{c}\text { Demonstrar a eficácia da } \\
\text { DLM no tratamento para } \\
\text { FEG, }\end{array}$ & $\begin{array}{l}\text { Verificou-se melhora do aspecto da } \\
\text { pele de todas as participantes da } \\
\text { pesquisa; e ambas referiram aumento } \\
\text { no débito urinário, principalmente nas } \\
10 \text { primeiras sessões. }\end{array}$ \\
\hline $\begin{array}{l}\text { Oliveira, } \\
2010\end{array}$ & $\begin{array}{l}\text { Drenagem linfática } \\
\text { manual aplicada em } \\
\text { gestantes. }\end{array}$ & $\begin{array}{c}\text { Verificar os efeitos da } \\
\text { DLM em gestante na } \\
\text { vigésima sétima semana } \\
\text { de gestação. }\end{array}$ & $\begin{array}{l}\text { Obteve melhor funcionamento } \\
\text { linfático, facilitação do retorno } \\
\text { circulatório, redução do edema e } \\
\text { relaxamento corporal. }\end{array}$ \\
\hline $\begin{array}{c}\text { Tacani, } \\
2010\end{array}$ & $\begin{array}{c}\text { Drenagem linfática } \\
\text { manual terapêutica } \\
\text { ou estética: existe } \\
\text { diferença? }\end{array}$ & $\begin{array}{c}\text { Apresentar } \\
\text { fundamentações gerais } \\
\text { sobre a DLM e verificar } \\
\text { semelhança nos } \\
\text { parâmetros de aplicação } \\
\text { da técnica para } \\
\text { finalidades terapêuticas e } \\
\text { estéticas. }\end{array}$ & $\begin{array}{c}\text { Após exposiçãa das técnicas e estudos } \\
\text { descritos, não foi identificado diferença } \\
\text { entre as técnicas de DLM terapêutica e } \\
\text { estética. }\end{array}$ \\
\hline $\begin{array}{l}\text { Silva; } \\
\text { Mejia, } \\
2012\end{array}$ & $\begin{array}{l}\text { Os benefícios da } \\
\text { drenagem linfática } \\
\text { no aspecto da pele } \\
\text { em mulheres com } \\
\text { fibro edema } \\
\text { gelóide. }\end{array}$ & $\begin{array}{l}\text { Analisar os benefícios da } \\
\text { DLM no aspecto da pele } \\
\text { em mulheres no FEG. }\end{array}$ & $\begin{array}{l}\text { Foi observado no estudo a técnica } \\
\text { desenvolvida apresenta melhora no } \\
\text { aspecto da pele no grau I e II. }\end{array}$ \\
\hline $\begin{array}{c}\text { Viana; } \\
\text { Maia, } 2013\end{array}$ & $\begin{array}{l}\text { Os efeitos da } \\
\text { drenagem linfática } \\
\text { manual no fibro } \\
\text { edema gelóide no } \\
\text { grau II }\end{array}$ & $\begin{array}{l}\text { Analisar os efeitos da } \\
\text { DLM no tratamento do } \\
\text { FEG no grau II. }\end{array}$ & $\begin{array}{l}\text { Os dados encontrados comprovam a } \\
\text { eficácia da DLM. Pois, a partir da } \\
\text { análise de estudos, foi identificado que } \\
\text { ambos os autores traziam opiniões } \\
\text { similares no que dizem sobre os efeitos } \\
\text { fisiológicos na FEG. }\end{array}$ \\
\hline
\end{tabular}




\begin{tabular}{|c|l|l|l|}
\hline $\begin{array}{c}\text { Soares et al., } \\
2015\end{array}$ & $\begin{array}{l}\text { Efeitos da drenagem } \\
\text { linfática manual } \\
\text { através da técnica de } \\
\text { Leduc no no } \\
\text { tratamento do fibro } \\
\text { edema gelóide. }\end{array}$ & $\begin{array}{l}\text { Identificar os efeitos da } \\
\text { de Leduc no tatamento } \\
\text { do FEG. }\end{array}$ & $\begin{array}{l}\text { O estudo apresentou remissão do edema } \\
\text { corporal, e consequente melhora no } \\
\text { quadro de FEG grau 1. Porém o fibro } \\
\text { edema gelóide grau 2, localizado nos } \\
\text { culotes, obteve apenas uma suave } \\
\text { melhora. }\end{array}$ \\
\hline
\end{tabular}

Fonte: Pesquisa do autor, 2018.

\section{Discussão}

A fisiopatologia exata do FEG deve ainda ser considerada uma questão em debate, embora a maioria dos pesquisadores concordem sobre o considerado em relação ao envolvimento da microcirculação reduzida e do edema intersticial. O sistema linfático encontrase paralelo ao sanguíneo. A sua função principal está em auxiliar o organismo a drenar o líquido intersticial, bem como remover resíduos celulares que o sistema sanguíneo não tem a capacidade de coletar (LEDUC, 2000; ROSSI, VERGNANINI, 2000; GUIRRO, GUIRRO, 2004; MIRRASHED et al 2004).

O estudo de Brandão, et al. (2010) mostraram o efeito da DLM no tratamento do FEG em mulheres. Foram realizadas 10 sessões de DLM em 10 mulheres sedentárias avaliadas com grau de I e III de FEG. Cada sessão tinha duração de 60 minutos, e foram realizados registros fotográficos das regiões glútea e das coxas. Os resultados comprovam satisfação das pacientes, onde foi visualizado melhora clínica no aspecto de pele, tanto através das fotografias quanto pela inspeção visual. Pois a DLM tem a finalidade de estimular o sistema linfático, eliminar resíduos metabólicos e toxinas, e reduzir o excesso de fluidos do corpo, o que justifica os resultados obtidos.

Além disso, Lima e Souza (2010) buscaram demonstrar a eficácia da DLM no FEG em 15 mulheres voluntárias entre 18 a 27 anos, sedentárias. As participantes foram avaliadas, e identificado o FEG como flácido, esse é um fator comum em indivíduos sedentários. Nesse estudo foi verificada a melhora do aspecto da pele de todas as participantes da pesquisa; e foi referido aumento no débito urinário, principalmente nas 10 primeiras sessões. No entanto, não pode ser observado resultados mais precisos, devido à ausência de participantes, e ganho de peso durante o tratamento. Os fatores citados contribuíram para aumento de retenção, e aparecimento de FEG. 
O estudo de Oliveira, (2010), buscou verificar os efeitos da DLM em uma gestante na vigésima sétima semana de gestação. O estudo descreve paciente gestante com 21 anos, na vigésima oitava semana de gestação; não possui vícios, e normalmente realiza caminhada por indicação médica; o atendimento foi realizado com paciente em decúbito lateral devido a gestação e não foi utilizado nenhum veículo, como o óleo. Após a primeira sessão de DLM, foi observado redução dos edemas nas regiões de mãos e pés. O estudo caracterizou-se com apenas duas sessões de drenagem linfática manual na paciente, e pôde notar-se de imediato a redução do edema principalmente nas regiões de mãos e pés. Devido à falta de interesse da paciente não pôde ser visto mais resultados.

Tacani (2010), apresentou fundamentações gerais sobre a DLM e verificou semelhança nos parâmetros de aplicação da técnica para finalidades terapêuticas e estéticas. Após analisar diversos estudos ficou evidente que, apesar de existir várias formas de desenvolver a técnica de DLM, e estas apresentarem aperfeiçoamento com o passar dos anos, ambas as técnicas tem a mesma finalidade e seguem os mesmos parâmetros para realização. Além disso, o estudo faz uma observação que relata existir outras terapias manuais como coadjuvantes e complementares para o tratamento de algumas disfunções estéticas, no entanto, devem respeitar a integridade dos tecidos manipulados e não podem ser denominadas como DLM.

Já no estudo de Silva e Mejia (2012), foi analisado os benefícios da DLM no aspecto da pele em mulheres com FEG. O estudo foi realizado com 3 participantes, onde cada uma apresentava um estágio de FEG específico. Foi realizado avaliação, e em seguida os protocolos de DLM foram desenvolvidas por dois meses, sendo 3 atendimentos semanais com duração de 30 minutos. A paciente com Grau I apresentou redução significativa de perimetria, bem como as alterações de pele. Já a paciente com grau II, os resultados foram moderados, e a de grau III não obteve resultados satisfatórios.

Em contrapartida, a aplicabilidade da DLM, traz resultados satisfatórios a paciente com FEG grau I e II, pois a melhora visual se deve ao fato da DLM promover a remoção do excesso do líquido presente no interstício. Por conseguinte, é necessário que haja mais estudos metodologicamente capazes de comprovar a eficácia da técnica quando realizada no grau III do FEG.

Viana e Maia (2013), buscaram evidenciar os efeitos da DLM no FEG no grau II. Os dados encontrados comprovam a eficácia da DLM. Pois, a partir da análise de estudos, foi 
identificado que ambos os autores traziam opiniões similares no que dizem sobre os efeitos fisiológicos na FEG.

Complementando o que foi dito, o estudo de Soares, et al. (2015), traz uma avaliação sobre os efeitos da DLM através da técnica de Leduc no tratamento do FEG. O estudo descreve uma paciente com índice de massa corporal elevada, 40 anos, sedentária, FEG nos graus I e II na região de culote, hábitos alimentares irregulares e ausência de atividade física. $\mathrm{O}$ estudo descreve que a participante manteve seus hábitos de vida normais e sedentarismo, e como resultado após o tratamento ocorreu remissão do edema corporal, e consequente melhora no quadro de FEG grau I. Porém o fibro edema gelóide grau II, localizado nos culotes, obteve apenas uma suave melhora. O FEG pode ocasionar dor e alteração funcional, além de influenciar a qualidade de vida. Além disso, o FEG deve ser tratado como um problema de saúde e não apenas como finalidade estética.

\section{Conclusão}

O FEG é uma disfunção estética que acomete tanto homens quanto mulheres. E atualmente é uma das queixas estéticas mais retratadas nos centros estéticos. A disfunção, além de acometer fisicamente e ser capaz de promover outras alterações, afeta a auto estima, bemestar e qualidade de vida do indivíduo.

Os estudos analisados comprovam a eficácia da DLM no tratamento do FEG em mulheres. No entanto, percebe-se também que não há um protocolo específico para o tratamento do FEG. Por se tratar de uma afecção multifatorial, e para se ter bons resultados, é preciso uma avaliação detalhada e capacitação profissional. Porém, observa-se que esta técnica deve ser associada a um conjunto de adaptações de hábitos de vida objetivando um melhor resultado da disfunção.

\section{Referências}

ABE, H.T.; FERREIRA, L.L. Tratamento Do Fibroedema gelóide Com Radiofrequência: revisão sistemática. Revista Pesquisa em Fisiot. Dez; Vol. 4, n.3: p. 206-214; 2014. 
ALMEIDA A.F.; BRANDÃO D.S.M.; SILVA J.C.; OLIVEIRA R.G.C.Q.; ARAÚJO R.C.; PITANGUI A.C.R. Avaliação do efeito da drenagem linfática manual e do ultrassom no fibroedema gelóide. Rev. bras. ciênc. saúde. 2011; Vol.9, n.28:31-7

BARROS, M. H. Fisioterapia: Drenagem Linfática Manual. São Paulo: Robe, 2001.

BAYRAKCI T. V.; AKBAYAK T.; BAKAR Y.; KAYIHAN H.; ERGUN N. Effects of mechanical massage, manual lymphatic drainage and connective tissue manipulation techniques on fat mass in omen with cellulite. J Eur Acad Dermatol Venereol; 2010.

BRANDÃO, D.S.M. et al. Avaliação da técnica de drenagem linfática manual no tratamento do fibro edema gelóide em mulheres. ConScientiae Saúde, Vol.9, n.4: p.618-624.; 2010.

BRITO J. Q. A; SILVA, A. P. O. Estudo de caso Sobre os Efeitos da Radiofrequência no tratamento do Fibro Edema Gelóide. Id online Rev. Psi. Vol.11, n. 35. ISSN 1981-1179.

COSTA, Adilson, et al. "Lipodistrofia ginóide e terapêutica clínica: análise crítica das publicações científicas disponíveis" Surgical \& Cosmetic Dermatology,Vol.4, n.1, 2012. Disponível em: http://www.redalyc.org/pdf/2655/265523678009.pdf

CUNHA, M. G, CUNHA, A. L. G, MACHADO, C. A. Fisiopatologia da lipodistrofia ginoide; Surgical \& Cosmetic Dermatology, vol. 7, num. 2, p. 98-102. Sociedade Brasileira de Dermatologia Rio de Janeiro, Brasil; 2015

ESTEVES R.M.; MARQUES F.; VIGATO A. Estudo comparativo dos efeitos do ultrassom e da endermologia no tratamento da lipodistrofia ginóide. Rev. Iniciação Científica UNINCOR; 2012.

FEDERICO M.R, GOMES S.V.C, MELO V.C, MARTINS R.B, LAURIA M.C, MOURA R.L et al. Tratamento de celulite (paniculopatia edemato fibroesclerótica) utilizando fonoforese com substância acoplante à base de hera, centella asiática e castanha da índia. Rev. Fisioter Ser.; Vol.1, n.1:p. 6-10; 2006

FERREIRA, J. B.; LEMOS, L. M. A.; SILVA, T. R. da. Qualidade de vida, imagem corporal e satisfação nos tratamentos estéticos. Ver. Pesquisa em Fisioterapia. Vol. 6; n,4:p. 402-410; nov/2016

FROIS, ERICA, MOREIRA, JACQUELINE, STENGEL, MARCIA. Mídias e a imagem corporal na adolescência: o corpo em discussão. Psicologia em estudo [em linea] 2011, 16 ( Maezo - Sin mês) : [Fecha de consulta: 29 de mayo de 2018]. Disponible em: http://www.redalyc.org/html/2871/287122137009/.

GALVÃO, M.M.M. Drenagem linfática manual e Ultra -Som no tratamento do fibro edema gelóide em região glútea: um estudo de caso. Faculdade Assis. Gurgacz, Cascavel: 2005.

GODOY J.M.P.; GODOY M.F.G. Celulite do diagnóstico ao tratamento. São José do Rio Preto: [S.n]; 2003. 
GRAVENA, B.P. Massagem de Drenagem Linfática no tratamento de Fibro Edema Gelóide em mulheres jovens. p. 11-59. Cascavel, 2004.

HEXEL, A.B.R.; Vergnanini, A.L. Cellulite: a review. J of the EuropAcad of Dermatol and venerol. Vol., n.4: p.251-262; jul/2000.

HEXEL, D.; Dalforno, T.O.; Gignachi, S. Definition, clinical aspects, associated conditions, and differential diagnosis. Cellulite Pathophysiology and treatment; basic and clinical dermatology, published in new York, 2006.

LAKATOS, E.M.; MARCONI, M.A. Fundamentos de metodologia científica. $5^{\mathrm{a}}$ ed. São Paulo: Atlas, 2003.

MACHADO G.C, VIEIRA R.B, OLIVEIRA N.M.L, LOPES C.R. Análise dos efeitos do ultrassom terapêutico e da eletrolipoforese nas alterações decorrentes do fibroedema gelóide. Fisioter. nov; Vol.24, n.3:471-9; 2011.

MATEUS, A. Caracterização do Fibro Edema Gelóide e Respectivos Tratamentos nos Estudantes de Fisioterapia. Inst. Politécnico de Lisboa Escola Superior de Tecnologia de Saúde de Lisboa. Mestrado em Fisioterapia. Lisboa, 2014.

MENEZES, R.C.; SILVA, S.G.; RIBEIRO, E.R. Ultra-som no Tratamento do Fibro Edema Gelóide. Rev Ins.; Vol.1, n. 1:p.10-14. jun-jul/2009.

NOOTHETI, P.K. A Single Center, Randomized, Comparative, Prospective Clinical Study to Determine the Efficacy of the VelaSmooth System versus the TriActive System for the Treatment of Cellulite. Las in Sur and Med. Vol.38: p. 908-912; 2006.

OLIVEIRA, N. Drenagem Linfática manual aplicada em gestante. Florianópolis. Trabalho apresentado ao Curso Técnico de Massoterapia da Escola de Massoterapia e terapias Naturais Fisio Vitae Ltda em Florianópolis/SC, 2010.

PETERSON, J.D.; GOLDMAN, M.P. Laser, Light, and Energy Devices for Cellulite and Lipodystrophy. Clin plastic surg.Vol.38: p.463-474; 2011.

RAWLINGS A.V. Cellulite and its treatment. Int J Cosmet Sci. Vol.28:175-90; 2006.

SCWARTZBACH, A.L.; TASSINARY, J.A.F. Drenagem Linfática Manual No Tratamento Do Fibro Edema Gelóide: Revisão Bibliográfica, 2011.

SILVA. E.M.; MEJIA, D.P.M. Os benefícios da drenagem linfática no aspecto da pele em mulheres com fibro edema gelóide. Trabalho de Pós-Graduação (Fisioterapia); Faculdade Cambury, 2012.

SILVA, R. M. V. et al. Avaliação do grau do fibro edema gelóide utilizando um sensor de infravermelho, Ver. Da Saúde e Biotecnologia. Vol 1; n,1:p. 18-30. Jul/Out, 2017. 
SOARES, N. S.; MIRANDA, A. C.H.; PRAÇA, L.R.; BASTOS, V.P.D.; MAIA, R.H.M.; VASCONCELOS, T.B.; Efeitos da drenagem linfática manual através da técnica de Leduc no tratamento do fibroedema gelóide: Estudo de caso. Rev. Saúde. Com; Vol. 11, n.2: p.156-161, 2015.

TACANI, R.; TACANI, Pascale. Drenagem Linfática Manual Terapêutica Ou Estética: Existe Diferença? Manual Lymphatic Drainage Therapeutic Or Aesthetic: Is There A Diference?. Rev. Bras. Ciên. Saúde/Revista de Atenção à Saúde. Vol.6, n.17; 2010.

VIANA, M.J.; MAIA, D.P. Os efeitos da drenagem linfática manual no fibro edema gelóide no grau II. Trabalho de Pós-Graduação (Fisioterapia); Faculdade Cambury, 2013.

VOLPI, A.A.A. Análise da eficácia da vacuoterapia no tratamento do fibro edema gelóide por meio da termografia e da biofotogrametria. Fisio Bra.; Vol.11, n.1; p.70-77; jan/fev, 2010.

GUSMÃO, Flávia F.; GUIMARÃES, Marcia M.; SILVA, Natália Cristina L.; FERREIRA, Juliana Barros. Efeito da drenagem linfática no tratamento do fibro edema gelóide em mulheres

\section{Como citar este artigo (Formato ABNT):}

GUSMÃO, Flávia F.; GUIMARÃES, Marcia M.; SILVA, Natália Cristina L.; FERREIRA, Juliana Barros. Efeito da drenagem linfática no tratamento do fibro edema gelóide em mulheres. Id on Line Rev.Mult. Psic., 2018, vol.12, n.40, p.1222-1231. ISSN: 1981-1179.

Recebido: 29/05/2018

Aceito 30/05/2018 